Supporting Information

\title{
Discovery of Ubiquitin Specific Protease 7 (USP7) Inhibitors with Novel Scaffold Structures by Virtual Screening, Molecular Dynamics Simulation, and Biological Evaluation
}

Shengjie Liu ${ }^{a}$, Xinyu Zhou ${ }^{a}$, Minglei Li ${ }^{\mathrm{a}}$, Wenfeng Zhao ${ }^{\mathrm{a}}$, Shuxi Zhou ${ }^{\mathrm{a}}$, Keguang Cheng ${ }^{\mathrm{b}}$, Qinglong Xu', Caiping Chen ${ }^{\mathrm{a}}$, Xiaoan Wen ${ }^{\mathrm{a}}$, Hongbin $\mathrm{Sun}^{\mathrm{a}}{ }^{*}$, Haoliang Yuan ${ }^{\mathrm{a}}{ }$

a Jiangsu Key Laboratory of Drug Discovery for Metabolic Disease and State Key Laboratory of Natural Medicines, China Pharmaceutical University, Nanjing 210009, P.R. China

${ }^{\mathrm{b}}$ State Key Laboratory for Chemistry and Molecular Engineering of Medicinal Resources, School of Chemistry and Pharmacy, Guangxi Normal University, 15 Yucai Road, Guilin 541004, P.R. China

*Corresponding Author.

*Email: hongbinsun@ @pu.edu.cn (H. S). Tel/Fax: +86-25-83271050.

*Email: yhl@cpu.edu.cn (H. Y). Tel/Fax: +86-25-83271050.

\section{Notes}

The authors declare no competing financial interest.

\section{Table of Contents}

Figure S1. USP7 inhibitory rates of compounds 14-24 at the concentration of 50

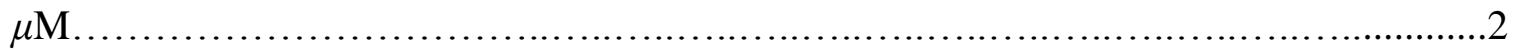

Figure S2. Results of PAINS filter for the 24 purchased compounds in this study..........3 Table S1. Specs IDNUMBERs and chemical structures of all the 24

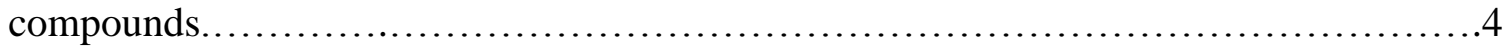




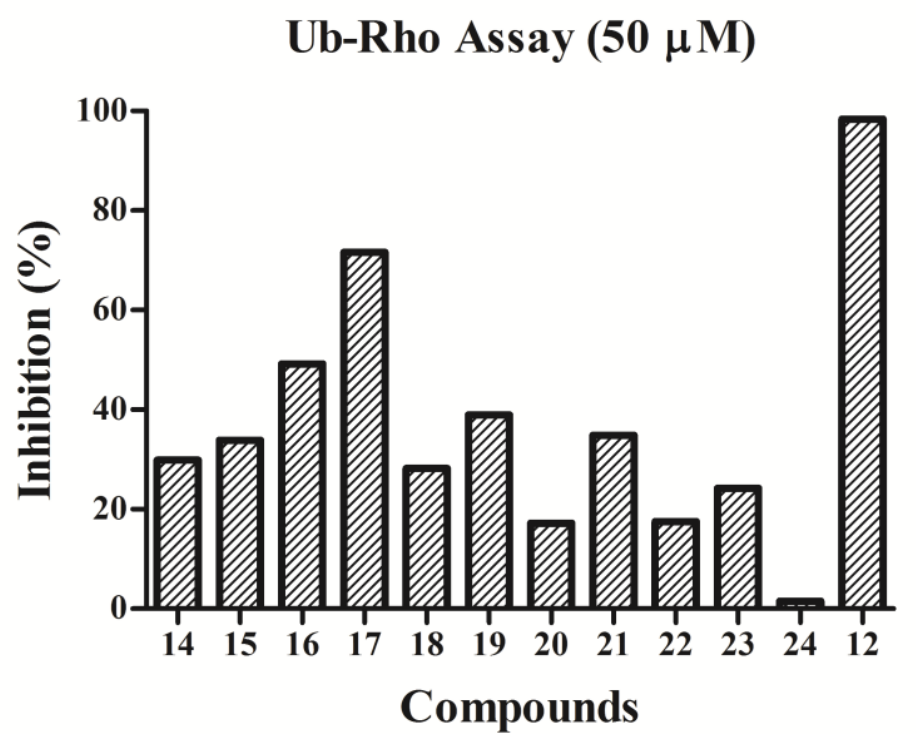

Figure S1. USP7 inhibitory rates of compounds 14-24 at the concentration of $50 \mu \mathrm{M}$. 

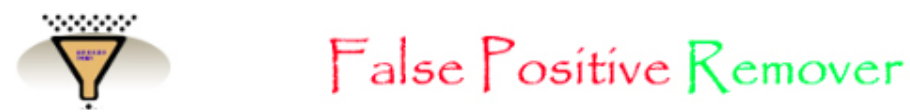

Welcome

Created 12-2009

Main Page $>$ > Retrieve Structure

Return

Totally 23 Compound(s) passed the filter.Download (Smiles format) view

Totally 1 Compound(s) were filtered out by the filter. Download (Smiles format). View Detailed The following are the structure(s) of compound(s) that are filtered out by the filter

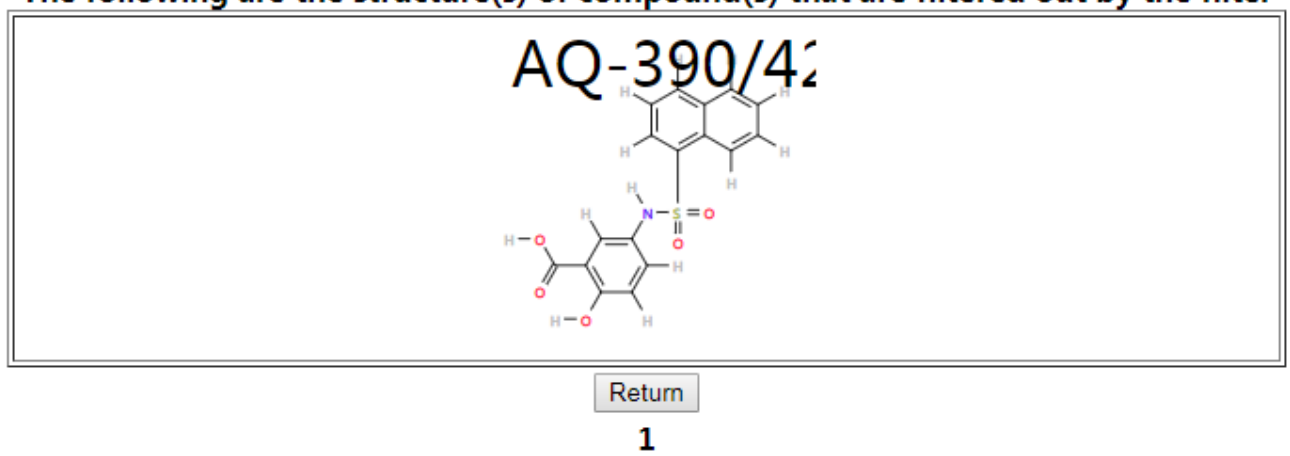

Figure S2. Results of PAINS filter for the 24 purchased compounds in this study. 
Table S1. Specs IDNUMBERs and chemical structures of all the 24 compounds.

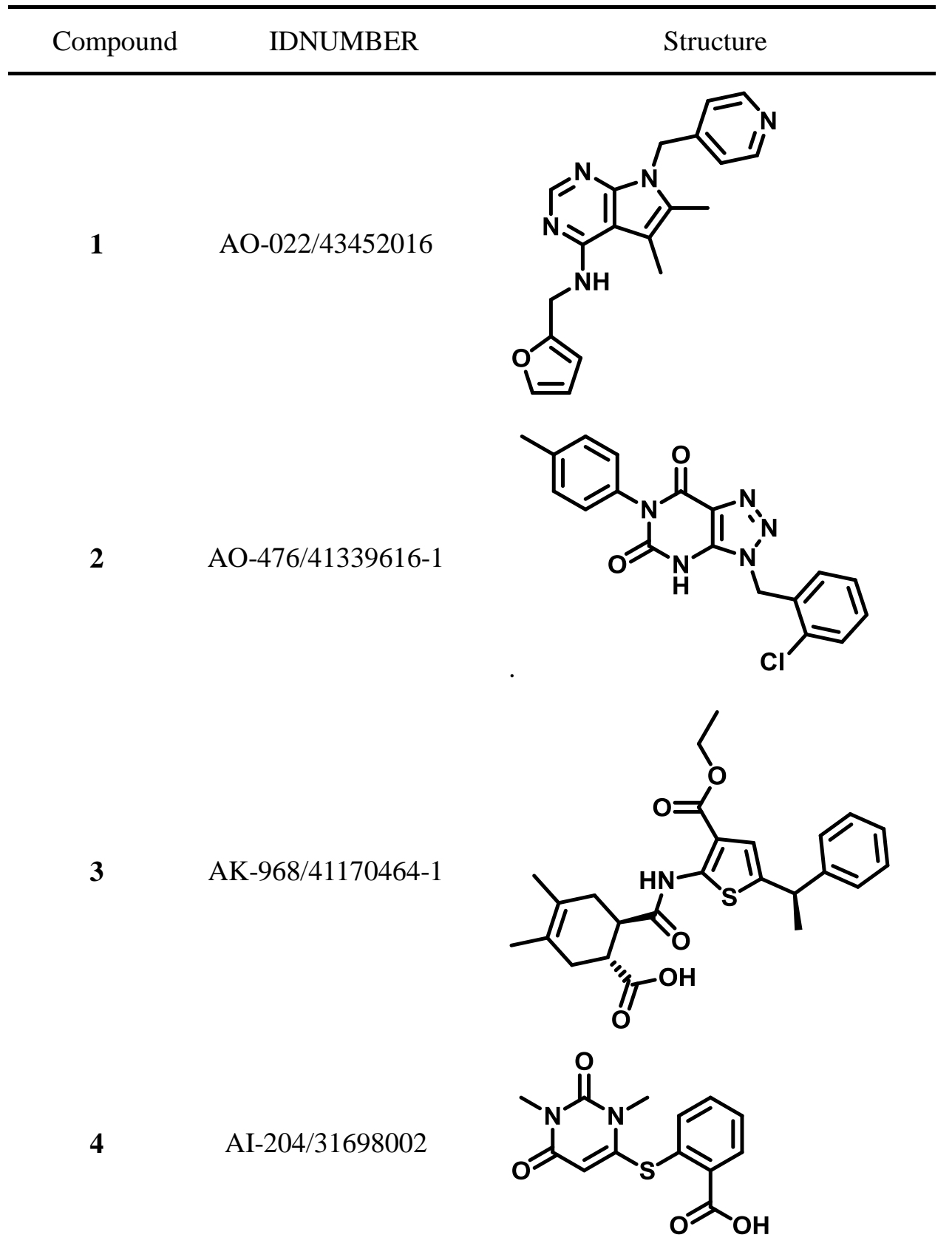


5

AK-918/12155010

6

7

AQ-390/42129431

AK-918/43446708

AG-690/40146935

8

9

AO-476/43417569
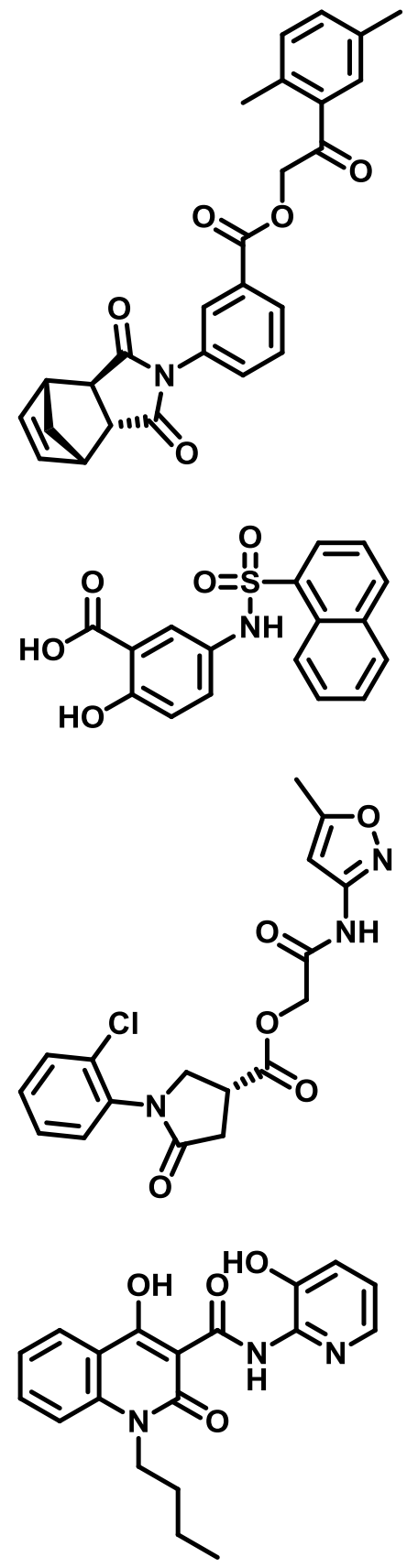<smiles>CC(=O)CSc1nc2[nH]ncc2c(=O)n1-c1ccccc1</smiles> 

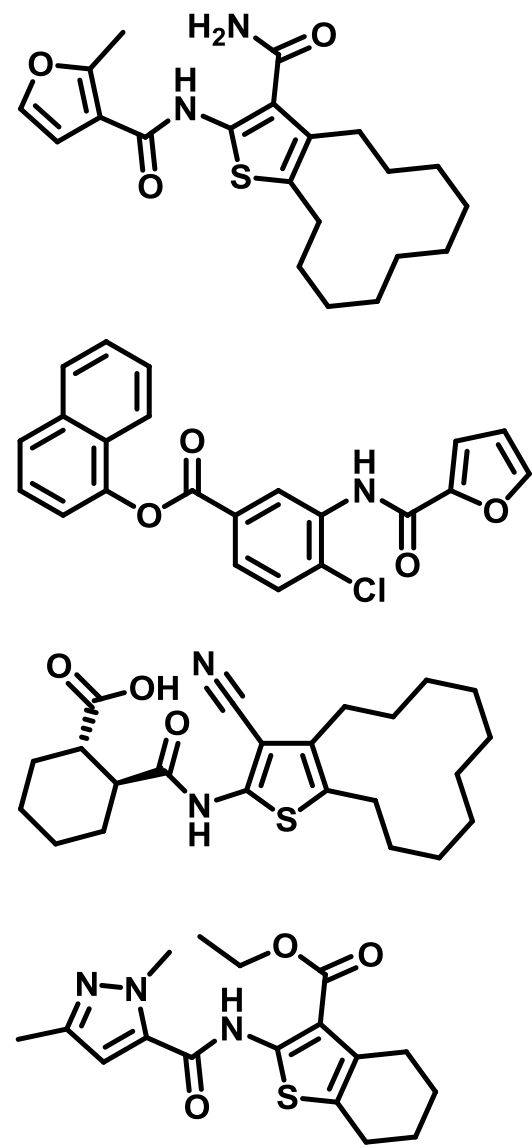

AK-968/12116004

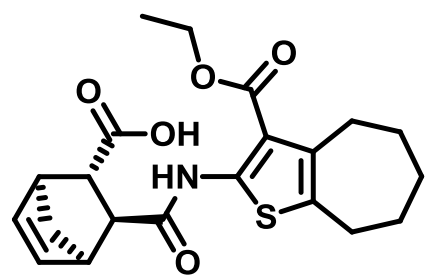

15

AK-968/41025074

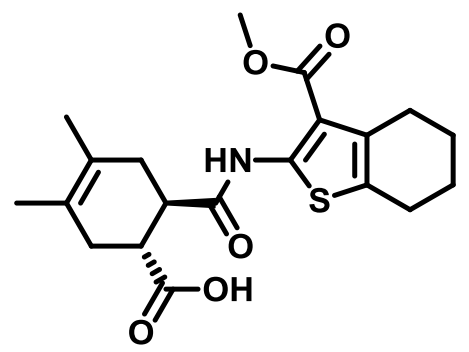

16

AK-968/14004736

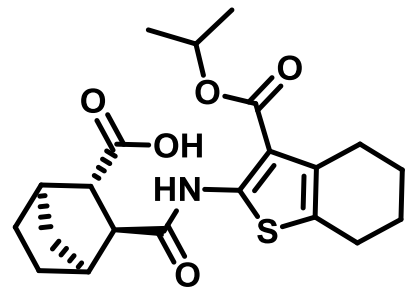




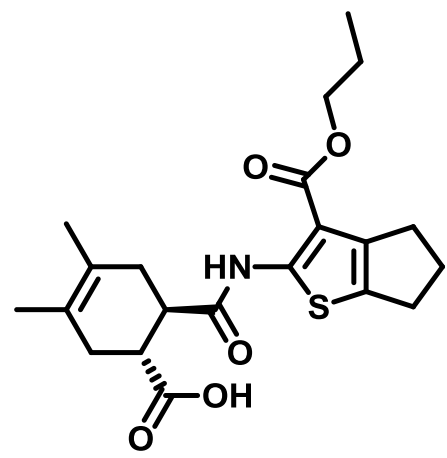

18

AK-968/37171093

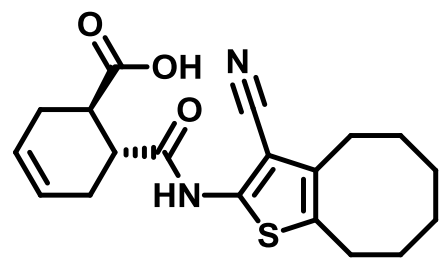

19

AK-968/40732357

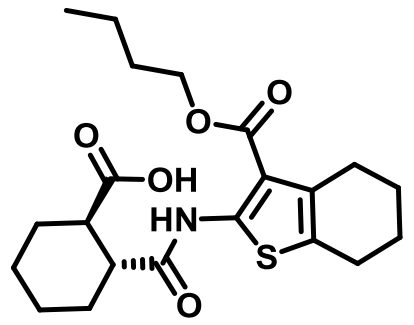

20

AK-968/11987433

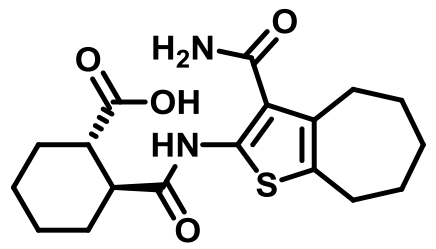

21

AK-968/37220043

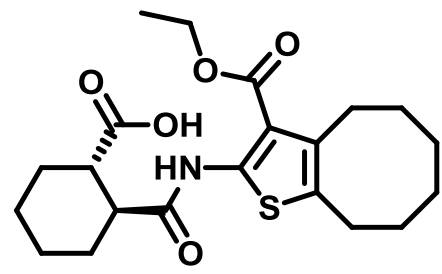

22

AK-968/41925316

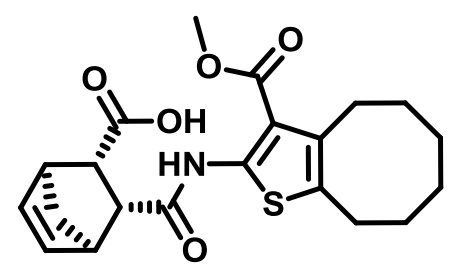




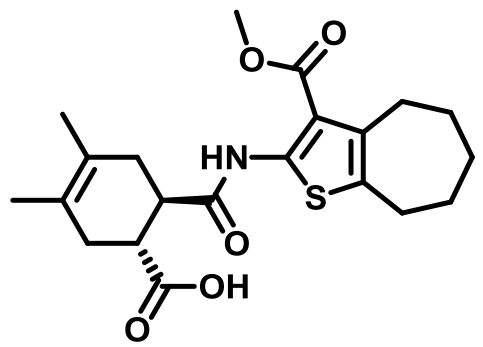

24

AK-968/36977292

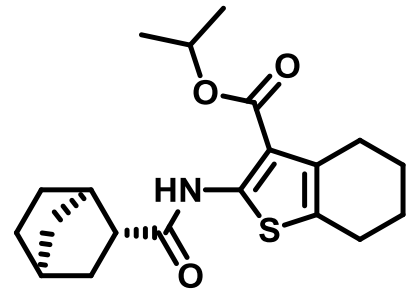

